\title{
Polarization speckles and generalized Stokes vector wave: a review [invited]
}

\author{
Takeda, Mitsuo; Wang, Wei; Hanson, Steen Grüner
}

Published in:

Proceedings of SPIE - The International Society for Optical Engineering

Link to article, DOI:

$10.1117 / 12.870928$

Publication date:

2010

Document Version

Early version, also known as pre-print

Link back to DTU Orbit

Citation (APA):

Takeda, M., Wang, W., \& Hanson, S. G. (2010). Polarization speckles and generalized Stokes vector wave: a review [invited]. Proceedings of SPIE - The International Society for Optical Engineering, 7387, 73870V. https://doi.org/10.1117/12.870928

\section{General rights}

Copyright and moral rights for the publications made accessible in the public portal are retained by the authors and/or other copyright owners and it is a condition of accessing publications that users recognise and abide by the legal requirements associated with these rights.

- Users may download and print one copy of any publication from the public portal for the purpose of private study or research.

- You may not further distribute the material or use it for any profit-making activity or commercial gain

- You may freely distribute the URL identifying the publication in the public portal

If you believe that this document breaches copyright please contact us providing details, and we will remove access to the work immediately and investigate your claim 


\title{
Polarization speckles and generalized Stokes vector wave: a review
}

\author{
Mitsuo Takeda*a ${ }^{*}$, Wei Wang ${ }^{b}$, Steen G. Hanson ${ }^{c}$ \\ ${ }^{a}$ UEC, Dept. of Engineering Science, The Univ. of Electro-Communications \\ 1-5-1, Chofugaoka, Chofu, Tokyo, 182-8585, Japan \\ ${ }^{b}$ Dept. of Mech. Engg., School of Engg. \& Phys. Sci., Heriot-Watt University \\ Edinburgh, EH14 4AS, United Kingdom \\ ${ }^{\mathrm{c} D T U}$ Fotonik, Department of Photonics Engineering \\ Technical University of Denmark, Dk-4000 Roskilde, Denmark
}

\begin{abstract}
We review some of the statistical properties of polarization-related speckle phenomena, with an introduction of a less known concept of polarization speckles and their spatial degree of polarization. As a useful means to characterize twopoint vector field correlations, we review the generalized Stokes parameters proposed by Korotkova and Wolf, and introduce its time-domain representation to describe the space-time evolution of the correlation between random electric vector fields at two different space-time points. This time-domain generalized Stokes vector, with components similar to those of the beam coherence polarization matrix proposed by Gori, is shown to obey the wave equation in exact analogy to a coherence function of scalar fields. Because of this wave nature, the time-domain generalized Stokes vector is referred to as generalized Stokes vector wave in this paper.
\end{abstract}

Keywords: Speckles, polarization, Stokes parameters, degree of polarization, coherence, stochastic process

\section{INTRODUCTION}

The term speckle patterns is usually associated with the fine-scale granular distribution of a light intensity pattern that arises from the interference of coherently superposed multiple random optical fields. Extensive studies have been made on their basic properties and applications ${ }^{1-3}$. In the majority of studies on speckle phenomena, these random optical fields have been treated as scalar optical fields, and the main interest has been in the statistical properties and applications of the intensity distribution of the speckle patterns, which we will call intensity speckles to distinguish them from polarization speckles to be introduced in this paper. Recently, statistical properties of random electric vector fields have come to attract new interest of researchers because of their importance in wide areas of practical applications such as biology ${ }^{4}$ and meteorology ${ }^{5}$. Statistical phenomena of random electric vector fields have relevance to the theories of speckles, polarization and coherence. Much effort is now being made by researchers to establish a new realm of statistical optics based on a unified theory on speckles ${ }^{3}$, coherence and polarization ${ }^{6}$. Clearly, it is far beyond the ability of the authors to cover these broad subjects. We will therefore restrict ourselves to the narrow-scope review on some of the statistical properties of polarization-related speckle phenomena, along with an introduction of a less known concept of polarization speckles and their spatial degree of polarization ${ }^{7}$. As a useful means to characterize two-point vector field correlations, we review the generalized Stokes parameters proposed by Korotkova and Wolf ${ }^{8}$. Then we introduce an alternative time-domain representation of the generalized Stokes parameter to describe the space-time evolution of the correlation between random electric vector fields at two different space-time points. This time-domain generalized Stokes vector has components similar to those of the beam coherence polarization matrix proposed by Gori ${ }^{9}$. We show that this time-domain generalized Stokes vector obeys the wave equation in exact analogy to a coherence function of scalar fields ${ }^{10}$. Because of this wave nature, it is referred to as generalized Stokes vector wave.

*takeda@ice.uec.ac.jp; phone +81 42443 5276; fax +81 424896072

Speckle 2010: Optical Metrology, edited by Armando Albertazzi Goncalves Jr., Guillermo H. Kaufmann, Proc. of SPIE Vol. 7387, 73870V · (c) 2010 SPIE · CCC code: 0277-786X/10/\$18 · doi: 10.1117/12.870928 


\section{POLARIZATION SPECKLES}

\subsection{What are polarization speckles?}

Figure 1 illustrates a conceptual scheme for generating polarization speckles. A linearly polarized fully coherent laser beam, with the vibration direction of electric field making an angle of 45 degrees to the $\mathrm{x}$-axis, enters a polarization beam splitter PBS. The incident beam is split into two mutually orthogonal linearly polarized beams, referred to as Xand Y-polarized beams, which impinge on moving random phase diffusers GG1 and GG2. The moving diffusers are assumed to be ideal statistically independent phase diffusers that impart a time-varying random phase to the orthogonally polarized beams without changing their state of polarization. In this model, the laser source is assumed to be ideally

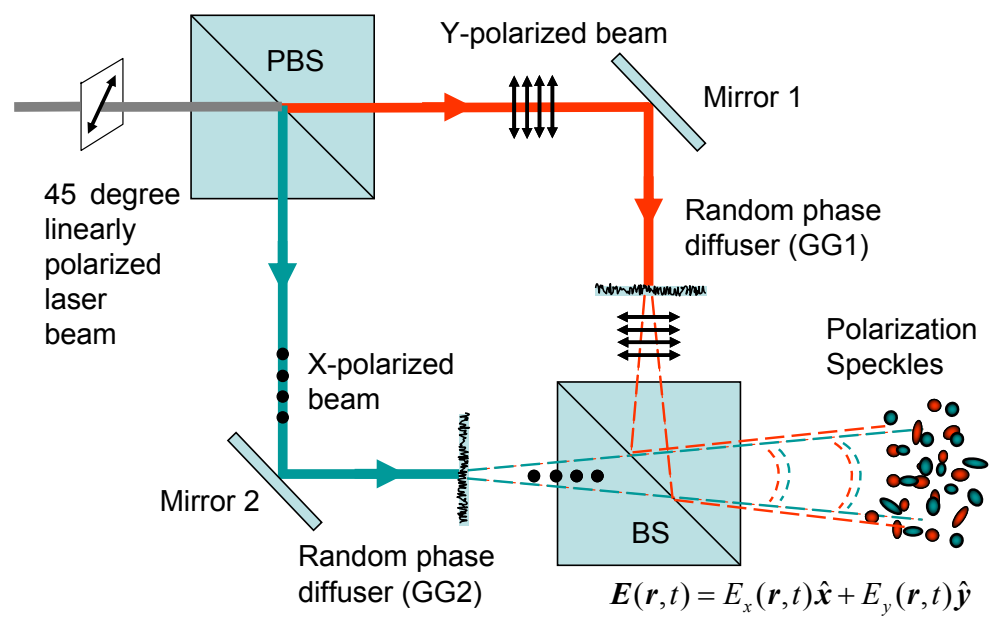

Figure 1. Conceptual scheme for generating polarization speckles.

stabilized so that the moving diffusers are the sole source that introduces a statistically random process into the system. The lights scattered from the moving random phase diffusers are combined by a non-polarization beam splitter BS to form a paraxial beam propagating in the z-direction. The scattered field at location $\boldsymbol{r}$ on the observation plane and at an instant $t$ is given by

$$
\boldsymbol{E}(\boldsymbol{r}, t)=E_{x}(\boldsymbol{r}, t) \hat{\boldsymbol{x}}+E_{y}(\boldsymbol{r}, t) \hat{\boldsymbol{y}},
$$

where $E_{x}(\boldsymbol{r}, t)$ and $E_{y}(\boldsymbol{r}, t)$ are x-and y-components of the scattered field, and $\hat{\boldsymbol{x}}$ and $\hat{\boldsymbol{y}}$ are unit vectors. If we detect the instantaneous intensity of the scattered field without using an analyzer, we have

$$
I(\boldsymbol{r}, t)=\boldsymbol{E}^{*}(\boldsymbol{r}, t) \cdot \boldsymbol{E}(\boldsymbol{r}, t)=\left|E_{x}(\boldsymbol{r}, t)\right|^{2}+\left|E_{y}(\boldsymbol{r}, t)\right|^{2},
$$

where we have assumed that the response time of the detector is much faster than the temporal fluctuation of the field. For those who are familiar with the high-speed fluctuation of natural light, this assumption may sound strange, but it does hold in our model, because, unlike the natural phase fluctuation in thermal light, we can realize arbitrarily slow phase fluctuations simply by reducing the speed of the moving diffusers. In other words, by using suitable random phase diffusers in slow motion, we can approximately perform a physical simulation for natural light in extremely slow motion with a greatly elongated time scale. The instantaneous intensity of Eq. (2), obtained by the polarization-blind detection scheme without using an analyzer, does not give any polarization-specific information other than the intensity superposition of the two time-varying intensity speckle patterns arising from the mutually orthogonal field components. However, if we detect the complex amplitudes of the two orthogonal field components, $E_{x}(\boldsymbol{r}, t)$ and $E_{y}(\boldsymbol{r}, t)$, by using an appropriate polarimetric interferometer, we can visualize spatio-temporal variations of the state of polarization. When we stop the movement of the diffusers, we will observe a static speckle-like random pattern that represents the spatial variation of the polarization state. We call this spatial random pattern of polarization states (which becomes visible only with a polarization-sensitive detection scheme) as a polarization speckle pattern to distinguish it from the conventional intensity speckle pattern observed by the polarization-blind detection scheme. Whereas the intensity speckle pattern can 
be characterized by only a single parameter of intensity $I$, the polarization speckle pattern needs to be characterized by e.g. a Stokes vector, which is composed of multiple Stokes parameters generally defined by

$$
\boldsymbol{S}(\boldsymbol{r} ; t)=\left(\begin{array}{c}
S_{0}(\boldsymbol{r} ; t) \\
S_{1}(\boldsymbol{r} ; t) \\
S_{2}(\boldsymbol{r} ; t) \\
S_{3}(\boldsymbol{r} ; t)
\end{array}\right)=\left(\begin{array}{c}
\left\langle E_{x}^{*}(\boldsymbol{r}, t) E_{x}(\boldsymbol{r}, t)\right\rangle+\left\langle E_{y}^{*}(\boldsymbol{r}, t) E_{y}(\boldsymbol{r}, t)\right\rangle \\
\left\langle E_{x}^{*}(\boldsymbol{r}, t) E_{x}(\boldsymbol{r}, t)\right\rangle-\left\langle E_{y}^{*}(\boldsymbol{r}, t) E_{y}(\boldsymbol{r}, t)\right\rangle \\
\left\langle E_{x}^{*}(\boldsymbol{r}, t) E_{y}(\boldsymbol{r}, t)\right\rangle+\left\langle E_{y}^{*}(\boldsymbol{r}, t) E_{x}(\boldsymbol{r}, t)\right\rangle \\
i\left[\left\langle E_{y}^{*}(\boldsymbol{r}, t) E_{x}(\boldsymbol{r}, t)\right\rangle-\left\langle E_{x}^{*}(\boldsymbol{r}, t) E_{y}(\boldsymbol{r}, t)\right\rangle\right]
\end{array}\right),
$$

where $\langle\ldots .>$ denotes the ensemble average. It is often the case that the ensemble average is replaced by the time average $\langle\ldots .\rangle_{\mathrm{T}}$ with the assumption that the field is stationary and ergodic in time. If both diffusers are statistically independent and have the same statistical property, then we have $S_{1}=S_{2}=S_{3}=0$ for sufficiently long averaging time or for sufficiently fast movement of the diffusers compared to the integration time of the detector. This situation is similar to the case of natural un-polarized quasi-monochromatic light except that the time scale is extremely elongated. In this case no polarization speckles can be observed. However, if the movement of the diffusers is stopped at time $t$, the fields become frozen at this instant of time, and have no temporal fluctuations afterwards. Then static polarization speckles manifest themselves because they are not averaged out by the time averaging operation. The Stokes parameters that describe such static polarization speckles are expressed by the instantaneous fields frozen at that particular instant of time $t$.

\subsection{Experimental generation and detection of polarization speckles}

Figure 2 shows an actual optical setup used for the generation and detection of polarization speckles. A polarimetric interferometer proposed by Oka and Ohtsuka ${ }^{11,12}$ was used for the detection of two orthogonal $\mathrm{x}$ - and $\mathrm{y}$-field components. Linearly polarized light from a He-Ne laser source was introduced into the Mach-Zehnder interferometer, and is subsequently divided into two components at a beam splitter BS1. The linearly polarized beam reflected at BS1 was collimated by a microscope objective (MO1) and Lens L1 to serve as a reference beam for interference. The collimated reference beam was split by a polarized beam splitter (PBS) into two orthogonal linearly polarized components. To balance the intensities of these two orthogonal components, a half-wave plate (HWP) was inserted before PBS to change the polarization plane of the incident beam. The reflected component passes through quarter-wave plate QWP1 to a tilted mirror (M2). When the beam is reflected, the tilt angle gives a spatial carrier frequency to the reflected beam. Similarly, the other beam reflected at mirror M3 is totally reflected at the PBS with the help of another quarter-wave plate QWP2. It follows that the reference beam from the PBS to beam splitter BS2 is composed of two orthogonal linearly polarized components with different spatial carrier frequencies. On the other hand, a depolarizer DP inserted in another arm of the interferometer depolarized the incident beam to function as a signal wave. By rotating the orientation angle of the depolarizer, we can control the spatial degree of polarization which will be defined in the next subsection. To adjust the size of polarization speckle, a $10 \times$ microscope objective $(\mathrm{MO} 2)$ was slid back and forth to produce a proper illumination spot size on a ground glass plate (GG), which scrambles the multi-polarization beam generated by DP. The generated

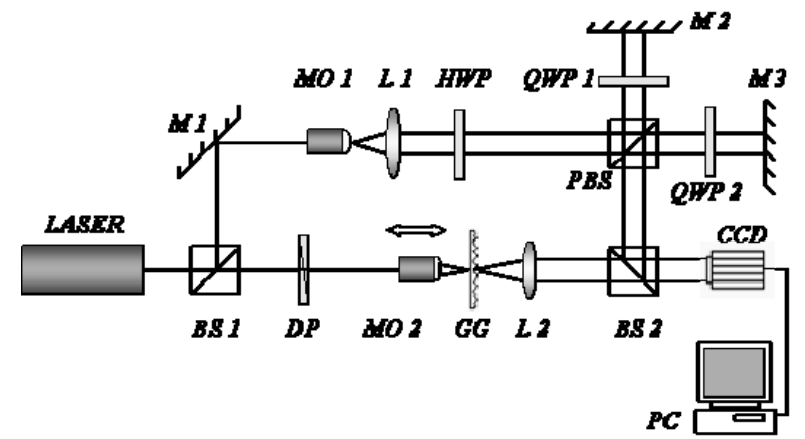

Figure 2. Experimental setup for detection of polarization speckles

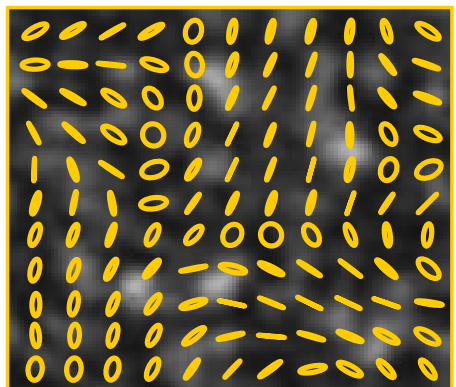

Figure 3. Example of polarization speckle pattern.

polarization speckle collimated by Lens L2 was made to interfere with the reference beam having two orthogonal linearly polarized components with different spatial carrier frequencies. The CCD camera records the interferogram of 
the polarization speckle in the far field. By applying the Fourier fringe analysis technique ${ }^{13}$ for two different spatial carrier frequencies, we recovered the electric field components from the two sets of fringes in the recorded interferogram, and obtained the distribution of polarization states which exhibits the polarization speckle. Figure 3 shows an example of the distribution of the polarization states obtained for a static ground glass. The spatial variation of the polarization states is indicated with the shape of polarization ellipses, with the intensity speckle pattern in the background. Each of the polarization domains, within which the polarization state remains effectively unchanged, may be regarded as an elementary unit of polarization speckles that constitutes the overall polarization speckle pattern.

\subsection{Temporal degree of polarization versus spatial degree of polarization}

In this subsection we will introduce a new concept of spatial degree of polarization, which is less familiar but seems to be suitable for the description of the characteristic of polarization speckles. Generally, the statistical properties of the two orthogonal field components are described by a $2 \times 2$ polarization matrix defined by

$$
\boldsymbol{J}(\boldsymbol{r}, t)=\left[\begin{array}{ll}
\left\langle E_{x}^{*}(\boldsymbol{r}, t) E_{x}(\boldsymbol{r}, t)\right\rangle & \left\langle E_{x}^{*}(\boldsymbol{r}, t) E_{y}(\boldsymbol{r}, t)\right\rangle \\
\left\langle E_{y}^{*}(\boldsymbol{r}, t) E_{x}(\boldsymbol{r}, t)\right\rangle & \left\langle E_{y}^{*}(\boldsymbol{r}, t) E_{y}(\boldsymbol{r}, t)\right\rangle
\end{array}\right] .
$$

It is also a standard procedure to define the degree of polarization by

$$
P=\left(\lambda_{1}-\lambda_{2}\right) /\left(\lambda_{1}+\lambda_{2}\right)=\left[1-4 \operatorname{det}(\boldsymbol{J}) /(\operatorname{tr}(\boldsymbol{J}))^{2}\right]^{1 / 2},
$$

where "tr" and "det" are the trace and determinant operation, respectively, and $\lambda_{1}$ and $\lambda_{2}$ are the real and nonnegative eigenvalues of the polarization matrix given by

$$
\lambda_{1,2}=\operatorname{tr}(\boldsymbol{J})\left[1 \pm \sqrt{1-4 \operatorname{det}(\boldsymbol{J}) /(\operatorname{tr}(\boldsymbol{J}))^{2}}\right] / 2 .
$$

As described before, it is often the case that the ensemble average $<\ldots .>$ in the polarization matrix is replaced by the time average $\left\langle\ldots .>_{\mathrm{T}}\right.$ with the assumption that the field is stationary and ergodic in time. The degree of polarization, defined by Eq. (6) for the polarization matrix $\boldsymbol{J}_{T}$ based on the time average, will be called temporal degree of polarization to distinguish it from spatial degree of polarization to be introduced in this paper. When the movement of the diffusers is stopped in order to observe polarization speckles, the fields become frozen to their states at the moment of the stop, and the time average operation on the elements of the polarization matrix in Eq. (5) will be removed. Therefore, we have

$$
\operatorname{det}\left(\boldsymbol{J}_{T}\right)=E_{x}^{*}(\boldsymbol{r}, t) E_{x}(\boldsymbol{r}, t) E_{y}^{*}(\boldsymbol{r}, t) E_{y}(\boldsymbol{r}, t)-E_{x}^{*}(\boldsymbol{r}, t) E_{y}(\boldsymbol{r}, t) E_{y}^{*}(\boldsymbol{r}, t) E_{x}(\boldsymbol{r}, t)=0,
$$

so that the temporal degree of polarization becomes unity $P_{T}=1$. This means that, when the diffusers are stopped, the

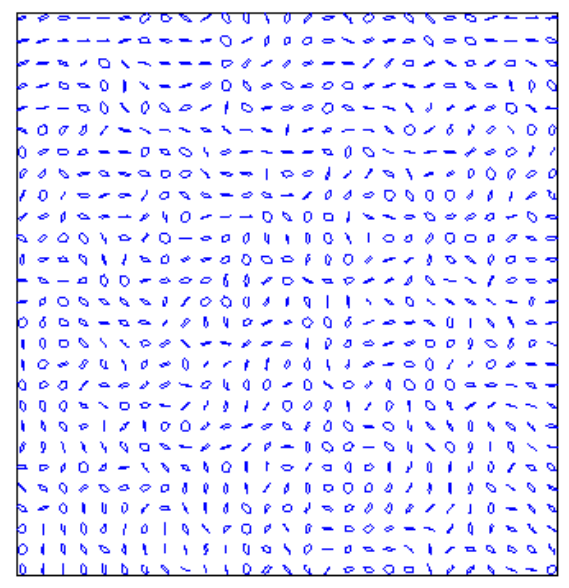

(a)

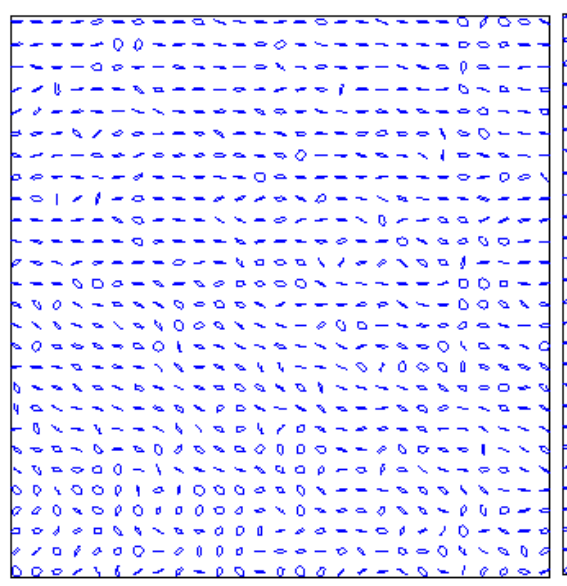

(b)

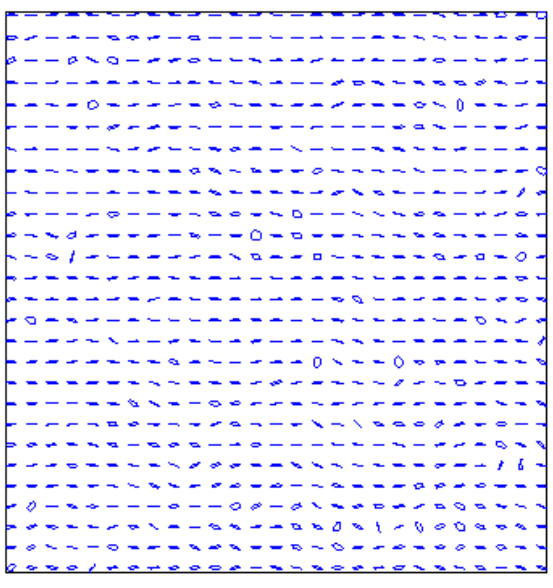

(c)

Figure 4. Mapping of polarization states for different spatial degrees of polarization for polarization speckles:

(a) $P_{s}=0.11$, (b) $P_{s}=0.28$, (c) $P_{s}=0.95$ 
fields become static and they are fully polarized according to the definition of temporal degree of polarization. Polarization speckles manifest themselves as spatial random variations of the state of polarization of such fully polarized static fields as shown in Fig. 3.

Our interest here is in the static random spatial distribution of the state of polarization of the fully polarized optical fields. To describe the statistical property of such fields, we introduce a less familiar concept of spatial degree of polarization. We replace the ensemble average $\left\langle\ldots .>\right.$ in the polarization matrix with the spatial average $\langle\ldots\rangle_{\mathrm{S}}$ with the assumption that the field is stationary and ergodic in space. Spatial degree of polarization $P_{S}$ is obtained from Eq. (6) by replacing the ensemble-average-based polarization matrix $\boldsymbol{J}$ with the spatial-average-based polarization matrix $\boldsymbol{J}_{S}$. Figure 4 shows an example of the polarization maps for the different spatial degree of polarization, which can be controlled by the rotation angle of the depolarizer in the experimental system shown in Fig.2. As seen from the figure, spatial degree of polarization is related to the degree of order or disorder of the spatial distribution of polarization states. One may note that spatial degree of polarization is conceptually analogous to the degree of macroscopic magnetization of magnetic materials induced by ordered or disordered orientations of many microscopic atomic dipoles, and polarization speckles bear similarity to magnetic domains in their geometrical structures.

\section{GENERALIZED STOKES VECTOR WAVE}

In the previous section, our focus was on polarization speckles in the static vector fields generated by the somewhat particular optical system composed of random phase diffusers and a stabilized laser source emitting fully polarized light. We now turn our attention to a more general case where the spatio-temporal variation of a stochastic electromagnetic beam is of main concern. The generalized Stokes parameters proposed by Korotkova and Wolf ${ }^{8}$ are particularly suitable for the description of the polarization properties of the stochastic electromagnetic beam. As a natural generalization of the spectral Stokes parameters, they defined the generalized Stokes parameters in the optical frequency domain for a pair of spectra (with frequency $\omega$ ) at two different spatial points $\left(\boldsymbol{r}_{1}, \boldsymbol{r}_{2}\right)$ :

$$
\boldsymbol{S}\left(\boldsymbol{r}_{1}, \boldsymbol{r}_{2} ; \omega\right)=\left(\begin{array}{c}
S_{0}\left(\boldsymbol{r}_{1}, \boldsymbol{r}_{2} ; \omega\right) \\
S_{1}\left(\boldsymbol{r}_{1}, \boldsymbol{r}_{2} ; \omega\right) \\
S_{2}\left(\boldsymbol{r}_{1}, \boldsymbol{r}_{2} ; \omega\right) \\
S_{3}\left(\boldsymbol{r}_{1}, \boldsymbol{r}_{2} ; \omega\right)
\end{array}\right)=\left(\begin{array}{c}
\left\langle E_{x}^{*}\left(\boldsymbol{r}_{1}, \omega\right) E_{x}\left(\boldsymbol{r}_{2}, \omega\right)\right\rangle+\left\langle E_{y}^{*}\left(\boldsymbol{r}_{1}, \omega\right) E_{y}\left(\boldsymbol{r}_{2}, \omega\right)\right\rangle \\
\left\langle E_{x}^{*}\left(\boldsymbol{r}_{1}, \omega\right) E_{x}\left(\boldsymbol{r}_{2}, \omega\right)\right\rangle-\left\langle E_{y}^{*}\left(\boldsymbol{r}_{1}, \omega\right) E_{y}\left(\boldsymbol{r}_{2}, \omega\right)\right\rangle \\
\left\langle E_{x}^{*}\left(\boldsymbol{r}_{1}, \omega\right) E_{y}\left(\boldsymbol{r}_{2}, \omega\right)\right\rangle+\left\langle E_{y}^{*}\left(\boldsymbol{r}_{1}, \omega\right) E_{x}\left(\boldsymbol{r}_{2}, \omega\right)\right\rangle \\
i\left[\left\langle E_{y}^{*}\left(\boldsymbol{r}_{1}, \omega\right) E_{x}\left(\boldsymbol{r}_{2}, \omega\right)\right\rangle-\left\langle E_{x}^{*}\left(\boldsymbol{r}_{1}, \omega\right) E_{y}\left(\boldsymbol{r}_{2}, \omega\right)\right\rangle\right]
\end{array}\right) .
$$

Note that implicit in this definition made in the frequency domain is that the fields are stationary in time.

To describe the space-time evolution of the correlation between random electric vector fields at two different space-time points, we introduce an alternative time-domain representation of the generalized Stokes parameters such that

$$
\boldsymbol{S}\left(\boldsymbol{r}_{1}, \boldsymbol{r}_{2} ; t_{1}, t_{2}\right)=\left(\begin{array}{c}
S_{0}\left(\boldsymbol{r}_{1}, \boldsymbol{r}_{2} ; t_{1}, t_{2}\right) \\
S_{1}\left(\boldsymbol{r}_{1}, \boldsymbol{r}_{2} ; t_{1}, t_{2}\right) \\
S_{2}\left(\boldsymbol{r}_{1}, \boldsymbol{r}_{2} ; t_{1}, t_{2}\right) \\
S_{3}\left(\boldsymbol{r}_{1}, \boldsymbol{r}_{2} ; t_{1}, t_{2}\right)
\end{array}\right)=\left(\begin{array}{c}
\left\langle E_{x}^{*}\left(\boldsymbol{r}_{1}, t_{1}\right) E_{x}\left(\boldsymbol{r}_{2}, t_{2}\right)\right\rangle+\left\langle E_{y}^{*}\left(\boldsymbol{r}_{1}, t_{1}\right) E_{y}\left(\boldsymbol{r}_{2}, t_{2}\right)\right\rangle \\
\left\langle E_{x}^{*}\left(\boldsymbol{r}_{1}, t_{1}\right) E_{x}\left(\boldsymbol{r}_{2}, t_{2}\right)\right\rangle-\left\langle E_{y}^{*}\left(\boldsymbol{r}_{1}, t_{1}\right) E_{y}\left(\boldsymbol{r}_{2}, t_{2}\right)\right\rangle \\
\left\langle E_{x}^{*}\left(\boldsymbol{r}_{1}, t_{1}\right) E_{y}\left(\boldsymbol{r}_{2}, t_{2}\right)\right\rangle+\left\langle E_{y}^{*}\left(\boldsymbol{r}_{1}, t_{1}\right) E_{x}\left(\boldsymbol{r}_{2}, t_{2}\right)\right\rangle \\
i\left[\left\langle E_{y}^{*}\left(\boldsymbol{r}_{1}, t_{1}\right) E_{x}\left(\boldsymbol{r}_{2}, t_{2}\right)\right\rangle-\left\langle E_{x}^{*}\left(\boldsymbol{r}_{1}, t_{1}\right) E_{y}\left(\boldsymbol{r}_{2}, t_{2}\right)\right\rangle\right]
\end{array}\right) .
$$

Note that this definition made in the time domain does not require the fields to be stationary in time or in space. In this sense this definition is more general than the spectrum domain representation in Eq. (9), although it has to pay the cost to include four parameters $\left(\mathbf{r}_{1}, \mathbf{r}_{2} ; t_{1}, t_{2}\right)$ rather than the three $\left(\mathbf{r}_{1}, \mathbf{r}_{2} ; \omega\right)$. Now let us show that this time-domain generalized Stokes vector obeys the wave equation in the same manner as the coherence function for scalar fields. The proof is rather straightforward. 
Let $E_{p}\left(\boldsymbol{r}_{m}, t_{m}\right)$ be the $p$ comportment of an optical field at position $\boldsymbol{r}_{m}$ and time $t_{m}$, with $p=x, y$ and $m=1,2$. Since each field component obeys the same wave equation in free space filled with a birefringence-free isotropic medium, we have

$$
\nabla_{m}^{2} E_{p}\left(\boldsymbol{r}_{m}, t_{m}\right)=\frac{1}{c^{2}} \frac{\partial^{2}}{\partial t_{m}^{2}} E_{p}\left(\boldsymbol{r}_{m}, t_{m}\right)
$$

Following a standard procedure found in textbooks on coherence theory ${ }^{2,10}$, we can readily see that the cross-correlation of the field components also obeys the same wave equation:

$$
\begin{aligned}
& \nabla_{m}^{2}\left\langle E_{p}^{*}\left(\boldsymbol{r}_{m}, t_{m}\right) E_{p^{\prime}}\left(\boldsymbol{r}_{m^{\prime}}, t_{m^{\prime}}\right)\right\rangle=\frac{1}{c^{2}} \frac{\partial^{2}}{\partial t_{m}^{2}}\left\langle E_{p}^{*}\left(\boldsymbol{r}_{m}, t_{m}\right) E_{p^{\prime}}\left(\boldsymbol{r}_{m^{\prime}}, t_{m^{\prime}}\right)\right\rangle \\
& \nabla_{m}^{2} \Gamma_{p p^{\prime}}\left(\boldsymbol{r}_{m}, \boldsymbol{r}_{m^{\prime}} ; t_{m}, t_{m^{\prime}}\right)=\frac{1}{c^{2}} \frac{\partial^{2}}{\partial t_{m}^{2}} \Gamma_{p p^{\prime}}\left(\boldsymbol{r}_{m}, \boldsymbol{r}_{m^{\prime}} ; t_{m}, t_{m^{\prime}}\right)
\end{aligned},
$$

where

$$
\Gamma_{p p^{\prime}}\left(\boldsymbol{r}_{m}, \boldsymbol{r}_{m^{\prime}} ; t_{m}, t_{m^{\prime}}\right)=\left\langle E_{p}^{*}\left(\boldsymbol{r}_{m}, t_{m}\right) E_{p^{\prime}}\left(\boldsymbol{r}_{m^{\prime}}, t_{m^{\prime}}\right)\right\rangle .
$$

Note that the generalized Stokes vector in Eq. (10) can be rewritten as

$$
\boldsymbol{S}\left(\boldsymbol{r}_{1}, \boldsymbol{r}_{2} ; t_{1}, t_{2}\right)=\left(\begin{array}{c}
S_{0}\left(\boldsymbol{r}_{1}, \boldsymbol{r}_{2} ; t_{1}, t_{2}\right) \\
S_{1}\left(\boldsymbol{r}_{1}, \boldsymbol{r}_{2} ; t_{1}, t_{2}\right) \\
S_{2}\left(\boldsymbol{r}_{1}, \boldsymbol{r}_{2} ; t_{1}, t_{2}\right) \\
S_{3}\left(\boldsymbol{r}_{1}, \boldsymbol{r}_{2} ; t_{1}, t_{2}\right)
\end{array}\right)=\left(\begin{array}{c}
\Gamma_{x x}\left(\boldsymbol{r}_{1}, t_{1} ; \boldsymbol{r}_{2}, t_{2}\right)+\Gamma_{y y}\left(\boldsymbol{r}_{1}, t_{1} ; \boldsymbol{r}_{2}, t_{2}\right) \\
\Gamma_{x x}\left(\boldsymbol{r}_{1}, t_{1} ; \boldsymbol{r}_{2}, t_{2}\right)-\Gamma_{y y}\left(\boldsymbol{r}_{1}, t_{1} ; \boldsymbol{r}_{2}, t_{2}\right) \\
\Gamma_{x y}\left(\boldsymbol{r}_{1}, t_{1} ; \boldsymbol{r}_{2}, t_{2}\right)+\Gamma_{y x}\left(\boldsymbol{r}_{1}, t_{1} ; \boldsymbol{r}_{2}, t_{2}\right) \\
i\left[\Gamma_{y x}\left(\boldsymbol{r}_{1}, t_{1} ; \boldsymbol{r}_{2}, t_{2}\right)-\Gamma_{x y}\left(\boldsymbol{r}_{1}, t_{1} ; \boldsymbol{r}_{2}, t_{2}\right)\right]
\end{array}\right) .
$$

Since the individual elements in the generalized Stokes vector obey a set of the same wave equations

$$
\begin{aligned}
& \nabla_{1}^{2} \Gamma_{p p^{\prime}}\left(\boldsymbol{r}_{1}, \boldsymbol{r}_{2} ; t_{1}, t_{2}\right)=\frac{1}{c^{2}} \frac{\partial^{2}}{\partial t_{1}^{2}} \Gamma_{p p^{\prime}}\left(\boldsymbol{r}_{1}, \boldsymbol{r}_{2} ; t_{1}, t_{2}\right) \\
& \nabla_{2}^{2} \Gamma_{p p^{\prime}}\left(\boldsymbol{r}_{1}, \boldsymbol{r}_{2} ; t_{1}, t_{2}\right)=\frac{1}{c^{2}} \frac{\partial^{2}}{\partial t_{2}^{2}} \Gamma_{p p^{\prime}}\left(\boldsymbol{r}_{1}, \boldsymbol{r}_{2} ; t_{1}, t_{2}\right)
\end{aligned}
$$

with regard to the two space-time points $\left(\boldsymbol{r}_{1}, t_{1}\right)$ and $\left(\boldsymbol{r}_{2}, t_{2}\right)$ while fixing the other, and since the generalized Stokes vector is composed of the linear superposition of such elements, the generalized Stokes vector also obeys the same set of wave equations:

$$
\begin{aligned}
& \nabla_{1}^{2} \boldsymbol{S}\left(\boldsymbol{r}_{\boldsymbol{l}}, \boldsymbol{r}_{2} ; t_{1}, t_{2}\right)=\frac{1}{c^{2}} \frac{\partial^{2}}{\partial t_{1}^{2}} \boldsymbol{S}\left(\boldsymbol{r}_{\boldsymbol{1}}, \boldsymbol{r}_{2} ; t_{1}, t_{2}\right) \\
& \nabla_{2}^{2} \boldsymbol{S}\left(\boldsymbol{r}_{\boldsymbol{l}}, \boldsymbol{r}_{2} ; t_{1}, t_{2}\right)=\frac{1}{c^{2}} \frac{\partial^{2}}{\partial t_{2}^{2}} \boldsymbol{S}\left(\boldsymbol{r}_{\boldsymbol{l}}, \boldsymbol{r}_{2} ; t_{1}, t_{2}\right)
\end{aligned}
$$

This result is not surprising at all, as we already know that the coherence function for scalar fields obeys the wave equation. However, by realizing the time-domain Stokes vector as a wave, we can gain a new insight into our understanding of various polarization-related coherence and speckle phenomena. For example, the implication of Eq. (16) is that we can introduce a concept of a generalized Stokes wave that behaves just like an optical field much in the same manner as does a conventional scalar coherence function. Once we reach this understanding, it is no surprise to see that the time-domain generalized Stokes wave obeys various physical laws, such as the van Cittert-Zernike theorem, which are already familiar to us with regard to scalar coherence theory. In fact, as far as the spectral-domain generalized Stokes parameters are concerned, such treatments can be found in not a few literatures ${ }^{6,14-16}$. 


\section{CONCLUSION}

We have presented a mini review on some of the statistical properties of polarization-related speckle and coherence phenomena, together with an introduction of a less known concept of polarization speckles and their spatial degree of polarization. Finally, we admit that, from the outset, we have abandoned the effort of presenting a well-balanced review which covers all the subjects relevant to the fields. We are also aware that many important papers are not included in the references. Interested readers may refer to Ref. 3 and Ref. 6 for a more comprehensive treatment of the subject.

\section{ACKNOWEDGMENT}

Part of this work was supported by Grant-in-Aid of JSPS B (2) No. 21360028.

\section{REFERENCES}

1. Dainty, J. C., Ed., [Laser Speckle and Related Phenomena], Springer-Verlag (1984).

2. Goodman, J. W., [Statistical Optics], Wiley-Interscience, New York (2000).

3. Goodman, J. W., [Speckle phenomena in Optics: Theory and Applications], Roberts-Company, Colorado (2006).

4. Angelsky, O. V., Ed. [Optical Correlation Techniques and Applications], SPIE Press, Washington, Chap.4 (2007).

5. Bringi, V. N., Hendry, A., "Technology of polarization diversity radars for meteorology," Radar in meteorology, American Meteorological Society, 153-190 (1990).

6. E. Wolf, [Introduction to the theory of coherence and polarization of light], Cambridge University Press, New York (2007).]

7. Wang, W., Hanson, S. G, and Takeda, M., "Statistics of polarization speckle: theory versus experiment," Proc. SPIE 7388, (03)1-9 (2009).

8. Korotkova, O. and Wolf, E., "Generalized Stokes parameters of random electromagnetic beams," Opt. Lett. 30, 198200 (2005).

9. Gori, F., "Matrix treatment for partially polarized, partially coherent beams," Opt. Lett., 23, 241-243 (1998).

10. Born, M. and Wolf, E., [Principle of Optics] Cambridge University Press, Cambridge, Chap. 10 (2005).

11. Oka, K. and Ohtsuka, Y., "Polarimetry for spatiotemporal photoelastic analysis," Exp. Mech. 33, 44-48 (1993).

12. Ohtsuka, Y. and Oka, K., "Contour mapping of the spatiotemporal state of polarization of light," Appl. Optics, 33(13), 2633-2636 (1994).

13. Takeda, M., Ina, H., and Kobayashi, S., "Fourier-transform method of fringe-pattern analysis for computer-based topography and interferometry," J. Opt. Soc. Am. 72, 156-160 (1982).

14. Wolf, E., "Correlation-induced changes in the degree of polarization, the degree of coherence, and the spectrum of random electromagnetic beams on propagation," Opt. Lett., 28, 1078-1080 (2003).

15. Gori, F., Santarsiero, M., R. Borghi, and Piquero, G., "Use of the van Cittert-Zernike theorem for partially polarized sources," Opt. Lett., 25, 1291-1293 (2000).

16. Hanson, S. G., Wang, W., Jakobsen, M. L., and Takeda, M., "Coherence and polarization of electromagnetic beams modulated by random phase screens and their changes through complex ABCD optical systems," J. Opt. Soc. Am. A, 25, 2338-2346 (2008). 